\title{
Über den Einfluß einzelner radioaktiver Elemente und Hormone anf die vasomotorische Erregbarkeit.
}

\author{
Von \\ Dr. K. T. A. Halbertsma. \\ (Aus dem Phyiologischen Laboratorium der Universität Utrecht \\ [Direktor: Prof. Dr. H. Zwaardemaker]). \\ Mit 2 Textabbildungen. \\ (Eingegangen am 18. Oktober 1922.)
}

Auf Anregung des Herrn Professor Dr. Zwaardemaker -- für dessen freundliche Hilfe und Ratschläge ich an dieser Stelle meinen verbindlichsten Dank ausspreche - habe ich eine Reihe von Untersuchungen ausgeführt über das Verhalten der vasomotorischen Erregbarkeit beim Frosche, falls man aus der Durchströmungsflüssigkeit das Kalium fortläßt und dieses Element von Uran, Thorium bzw. Rubidium ersetzt ${ }^{1}$ ). Außerdem ist der Einfluß einzelner Hormone (Adrenalin, Histamin und Cholin) auf die soeben genannten Wirkungen untersucht worden.

\section{Methodik.}

Beim Läwen-Trendelenburgpräparat ${ }^{2}$ ) strömte die Flüssigkeit durch eine Glaskanüle in die Aorta abdominalis. In allen großen Exemplaren von $R$. temporaria und $R$. esculenta, die wir durcheinander verwandten, tropfte die Durchströmungsflüssigkeit aus einer Kanüle (in die V. abdominalis) ab. Das Reservoir bestand aus 3 Mariotte schen Flaschen zu je 2 l. Sie waren mittels drei Glasröhren, die sich später vereinigten, mit dem zuführenden Gummischlauch des Präparates verbunden. Durch einen Hahn, der in jedem Glasrohr angebracht war, konnte man schnell die erwünschte Flüssigkeit zutreten lassen.

Die verwendete Ringerlösung ohne Kalium war folgendermaßen zusammengestellt: auf $1000 \mathrm{Aq}$. pur. fanden sich $6,5 \mathrm{~g} \mathrm{NaCl} 0,200 \mathrm{~g} \mathrm{CaCl}_{2}$, $0,200 \mathrm{~g} \mathrm{NaHCO}_{3}$. Sie befand sich in allen Versuchen in einer der drei Flaschen. In einer der zwei anderen befand sich diese Flüssigkeit, wozu jedoch radioaktives Element gefügt worden war. Die dritte Flasche enthielt eineLösung des zu untersuchenden Stoffes in kaliumloser Ringerlösung. In allen Versuchsreihen wurde mit Durchströmung mittels kaliumloser Ringerlösung angefangen; diese dauerte ungefähr $1 / 2$ Stunde; dann strömte die Flüssigkeit klar aus. Daran schloß sich die Durch-

I) Im Utrechtschen physiologischen Laboratorium wurde gezeigt, daß a) $\mathrm{Ka}$ lium sei in einer Reihe von Systemen und Funktionen von anderen radioaktiven Elementen in äquiradioaktiven Mengen ersetzbar; b) die Wirkung des Kaliums oder Rubidiums werde, mit anderen radioaktiven Elementen ( $\alpha$-Strahlern) zusammengebracht, von diesen letzten in bestimmten Mengen aufgehoben (Klin. Wochenschr. 1, Nr. 11; Ergebn. d. Physiol. 1921, S. 326).

2) Pflügers Arch. f. d. ges. Physiol. 103, 476. 1904. 
strömung mit kaliumhaltiger Ringerlösung an, und nachher wieder die mit kaliumloser Ringerlösung. Dann wurde die Wirkung des zu untersuchenden Stoffes geprüft. Die einzelnen Durchströmungen dauerten ungefähr 1/4 Stunde, das ganze Experiment höchstens 4-6 Stunden.

Der Durchströmungsdruck beträgt durchschnittlich $20-25 \mathrm{~cm} \mathrm{H}_{2} \mathrm{O}$.

Die Durchströmungsgeschwindigkeit wurde mittels Zählung der in der Zeiteinheit abfließenden Tropfen (aus der Venenkanüle) festgestellt. Dies geschah vermittels einer Uhr oder eines Tropfenzählapparates ${ }^{1}$ ), das die Zahl der gefallenen Tropfen auf einem rotierenden Zylinder registrierte. Weil der Druck konstant war, wurde aus einer Verminderung der Tropfenzahl in der Zeiteinheit - in unserem Falle in der Minute eine Verengerung, aus der Vermehrung dieser Zahl eine Erweiterung der durchströmten Gefäßen abgeleitet.

Auf Grund meiner Erfahrung habe ich diese Methode zur Bestimmung der Gefäßweite der mikroskopischen vorgezogen.

Die Wasserstoffionen-Konzentration der angewandten Flüssigkeiten schwankte je nach dem Alter der in den Glasbehältern aufbewahrten Lösungen. Im Mittel fand ich $p_{\mathbf{H}}=8$; bei Uranzufügung näherte man sich einigermaßen der Acidität, jedoch wenn physiologische Mengen Verwendung fanden in unbedeutendem Maße. Dieselbe Durchströmungsflüssigkeit wurde höchstens 2-3 Tage nacheinander verwendet. Die Reizung des N. ischiadicus geschah mittels Platinelektroden (48 Induktionsschläge pro Sekunde), jedesmal $1 / 2$ Minute links und rechts sofort nacheinander. Die Spulendistanz des Induktoriums wurde jedesmal so gewählt, daß bei Schließung des faradischen Stromkreises keine sichtbaren Muskelzuckungen am betreffenden Hinterbein auftraten. Zwischen zwei aufeinander folgenden Reizungen wurde mindestens 5 Minuten gewartet.

\section{Ergebnisse. \\ A. Bei alleiniger Durchströmung.}

Gunzburg ${ }^{2}$ ) hat uns gelehrt, daß Durchströmung der Froschgefäße mit kaliumloser Ringerlösung in einigen Stunden Ödem zum Vorschein treten läßt; durch rechtzeitige Zufügung geeigneter Mengen Kalium, Rubidium, Uran oder Thorium ist dies zu verhindern. Bei meinen Versuchen hatte ich selbstverständlich diesen Ergebnissen Rechnung zu tragen.

Von den $\alpha$-Strahlern untersuchte ich Uran (und zwar Uranylnitrat bzw. -sulfat pro miscue) und Thoriumnitrat.

Die Lösungen dieses letzten Salzes wurden jedesmal frisch hergestellt, weil sich meistens innerhalb einiger Stunden ein deutliches Präcipitat von $\mathrm{ThO}_{2}$ in der Flüssigkeit formt. Außerdem wurde die ThoriumRingerlösung stets mittels $\mathrm{Na}_{2} \mathrm{CO}_{3}$ sorgfältig neutralisiert; das Thorium-

1) Arch. néerland. de physiol. 2, 534. 1918.

2) Arch. Neerl. de Physiol. T. IV. 1918, S. 364. 
nitrat würde nämlich sonst in den von mir angewandten Konzentrationen der Lösung eine saure Reaktion verliehen haben.

Bei den untersuchten Dosen $\left(5-20 \mathrm{mg} \mathrm{Th}\left(\mathrm{NO}_{3}\right)_{4}\right.$ pro Liter) entwickelte sich stets allmählich Ödem.

Das Uran verhielt sich ungefähr ebenso : bei einer Dosis von $0-350 \mathrm{mg}$ Uransalz pro Liter trat immer Ödem zum Vorschein.

Als Vertreter der $\beta$-Strahlen wurden Kalium und Rubidium zur Untersuchung ausgewählt.

War die Kaliumdosis klein (10-20 $\mathrm{mg} \mathrm{KCl}$ pro Liter), so entwickelte sich allmählich ein mäßiges Ödem; bei Anwendung von $200 \mathrm{mg} \mathrm{KCl}$ pro Liter und höheren Dosen nahm das sich entwickelnde Ödem nicht so schnell $\mathrm{zu}$, wie es bei den niedrigen Dosen im allgemeinen der Fall war.

Durchströmung mit $250 \mathrm{mg}$ RbJ pro Liter ließ während 2-3 Stunden die Anzahl der herausfließenden Tropfen konstant bleiben; bei Anwendung von kleineren Dosen entstand jedesmal allmählich Ödem.

Weil, wie Gunzburg (1. c.) zeigte, bei Durchströmung mit Ringerlösung ohne Kalium, stets eine bestimmte Menge dieses Stoffes in der Ausströmungsflüssigkeit anwesend ist, habe ich das Gehalt der Ausströmungsflüssigkeit an Kalium, das in diesem Falle aus den die Gefäße umringenden Geweben stammen soll, in geeigneten Versuchen zu bestimmen versucht.

Die Resultate, mit der Methode de Koninck ${ }^{1}$ )-Bousser ${ }^{2}$ ) erhalten, sind aus nachstehender Tabelle ersichtlich (Flüssigkeit aus der V. abdominalis aufgefangen).

\begin{tabular}{|c|c|c|c|}
\hline Jahreszeit & $\begin{array}{l}\text { Anzahl } \\
\text { Stunden } \\
\text { Durch- } \\
\text { strömung }\end{array}$ & $\begin{array}{c}\text { mg KCl } \\
\text { pro } \\
\text { Liter }\end{array}$ & $\begin{array}{l}\text { Durchschnitt- } \\
\text { liche AustuB- } \\
\text { menge Kalium } \\
\text { in } 6 \text { Stunden }\end{array}$ \\
\hline \multirow{4}{*}{ Sommer 1918} & 1 & 16 & \multirow{4}{*}{20,5} \\
\hline & 2 & 22 & \\
\hline & 4 & 28 & \\
\hline & 6 & 16 & \\
\hline \multirow{4}{*}{ Herbst 1918.} & 1 & 20 & \multirow{4}{*}{23,75} \\
\hline & 2 & 25 & \\
\hline & 4 & 28 & \\
\hline & 6 & 22 & \\
\hline \multirow{4}{*}{ Winter $1918 / 19$} & 1 & $2 I$ & \multirow{4}{*}{23,25} \\
\hline & 2 & 20 & \\
\hline & 4 & 28 & \\
\hline & 6 & 25 & \\
\hline \multirow{4}{*}{ Frühjahr 1919} & 1 & 16 & \multirow{4}{*}{18,5} \\
\hline & 2 & 20 & \\
\hline & 4 & 22 & \\
\hline & 6 & 16 & \\
\hline
\end{tabular}

1) Zeitschr. f. analyt. Chem. 1891, Nr. 20, S. 390.

2) Journ. of the Americ. chem. soc. 1912, S. 1567. 
614 K. T. A. Halbertsma: Über den Einfluß cinzelner radioaktiver Elemente

Zwei Tatsachen gehen hier hervor:

1. Bei Durchströmung mit kaliumloser Ringlösung sind die Durchschnittsmengen gelösten Kaliums in der Ausströmungsflüssigkeit im Frühjahr und Sommer kleiner als im Herbst und Winter.

2. Die Durchschnittsmengen gelösten Kaliums in der Durchströ. mungsflüssigkeit nehmen in den ersten 4 Stunden der Durchströmung zu; nachher nehmen sie wieder ab.

\section{B. Ergebnisse der Nervenreizung.}

Im allgemeinen wurde an bestimmten Zeitpunkten $10 \mathrm{bzw} .20$ bis 30 Minuten nach Anfang der Durchströmung gereizt. Bei anfänglicher Durchströmung mit kaliumloser Ringerlösung ergab sich, daß die Vasoconstrictoren am meisten reizbar sind.

Experiment Nr. 1. $5^{\text {h }} 23^{\prime}$. Anfang der Durchströmung; Tropfenzahl pro Minute $=28$.

$5^{\text {h }} 33^{\prime}$. Nervenreizung (Spulendistanz $\left.=14,5 \mathrm{~cm}\right)$. Tropfenzahl pro Minute unmittelbar vor der Reizung $=28$. Tropfenzahl pro Minute unmittelbar nach der Reizung $=26$.

$5^{\text {h }} 43^{\prime}$. Nervenreizung (Spulendistanz $=14,5 \mathrm{~cm}$ ). Tropfenzahl pro Minute unmittelbar vor der Reizung $=\mathbf{2 6}$. Tropfenzahl pro Minute unmittelbar nach der Reizung $=24$.

Derselbe Befund ergab sich bei Durchströmung mit Kaliumringer (100 mg KCl pro Liter).

Experiment Nr. 2. $4^{\text {h }} 10^{\prime}$. Anfang der Durchströmung; Tropfenzahl pro Minute $=50$.

$4^{\text {h }} 20^{\prime}$. Nervenreizung (Spulendistanz $=14,5 \mathrm{~cm}$ ). Tropfenzahl pro Minute unmittelbar vor der Reizung $=50$.

Tropfenzahl pro Minute unmittelbar nach der Reizung $=40$.

$4^{\mathrm{h}} 30^{\prime}$. Nervenreizung (wie um $4^{\mathrm{h}} 20^{\prime}$ ). Tropfenzahl pro Minute unmittelbar vor der Reizung $=40$. Tropfenzahl unmittelbar nach der Reizung pro Minute $=30$.

In einer Reihe von Versuchen, wo stets mit Kaliumringer von wechselndem Gehalt durchströmt wurde, blieb die vasomotorische Reizbarkeit stets im selben Sinne erhalten.

Weil die Möglichkeit vorlag, daß diese Reizbarkeit von dem aus den Geweben herstammenden Kalium erhalten wurde, wandte ich den Kunstgriff an, der im Utrechtschen physiologischen Laboratorium gebraucht wird in dem Falle, wenn der Stillstand des künstlich durchströmten Froschherzen allzulange auf sich warten läßt. Zufügung einer Spur Uransalz zur Ringerlösung ohne Kalium hat dann oft sofort den Stillstand zur Folge. Dieser Kunstgriff war auch in meinem Falle imstande die vasomotorische Reizbarkeit zum Schwinden zu bringen: die faradische 
Reizung konnte jetzt nicht mehr die Zahl der in der Minute abfließenden Tropfen abändern.

Die Vasomotoren waren also bei Anwendung von Ringerlösung ohne Kalium, wozu eine Spur Uran gefügt worden war, unreizbar.

Experiment Nr. 3. $4^{\mathrm{h}} 00^{\prime}$. Anfang der Durchströmung (Ringerlösung ohne Kalium $+3 \mathrm{mg}$ Uransalz pro Liter).

$4^{\mathrm{h}} 15^{\prime}$. Nervenreizung (Spulendistanz $=28 \mathrm{~cm}$ ). Tropfenzahl pro Minute unmittelbar vor und nach der Reizung $=23$.

$4^{\mathrm{h}} 30^{\prime}$. Nervenreizung (wie um $4^{\mathrm{h}} 15$ ). Tropfenzahl pro Minute unmittelbar vor und nach der Reizung $=23$.

Die anzuwendende Menge des Uransalzes variiert mit den Jahreszeiten: im Winter braucht man 2-4 mg pro Liter, im Sommer genügen 2-3 mg pro Liter. Wenn man diesem Gemisch kaliumloser Ringerlösung mit einer Spur Uransalz, das ich seiner besonderen Eigenschaft wegen „Nullgemisch“ genannt habe, eine bestimmte Menge radioaktiven (Kalium-, Rubidium-, Uran-, Thorium-) Salzes zufügt, so kehrt sofort die verschwundene vasomotorische Erregbarkeit wieder zurück, und bleibt, wenn man nachher immer wieder neue Mengen eines bestimmten Salzes zufügt, innerhalb gewissen Grenzen erhalten.

Das ,erregbare“ Gebiet schwankt für die vier Metalle in verschiedenen Grenzen. Für das Uran liegt es zwischen 5 und $40 \mathrm{mg}$ Uransalz pro Liter (Winterdosis); für das Thorium zwischen 5 und $20 \mathrm{mg}$ Thoriumnitrat pro Liter (Sommerdosis).

Fügt man $5 \mathrm{mg} \mathrm{Th}\left(\mathrm{NO}_{3}\right)_{4}$ pro Liter dem Nullgemisch zu, so ändert sich nach der ersten Reizung (15 Minuten nach Anfang der Durchströmung) die Tropfenzahl nicht, bei der zweiten Reizung (15 Minuten später) vermindert es; bei den höchsten angewandten Th-Dosen zeigte sich das umgekehrte. Für das Uran konnte ein derartiges Verhalten nicht gezeigt werden, d. h. bei Anwendung der eben angedeuteten Minimumbzw. Maximumdosis; hier kehrte, ebenso wie bei den übrigen angewandten Th-Dosen die Erregbarkeit ohne weiteres sofort zurück.

Die „Erregbarkeitsgrenzen“ liegen für die $\beta$-Strahler viel weiter auseinander; für das Kalium zwischen 10 und $350 \mathrm{mg} \mathrm{KCl}$ pro Liter, für das Rubidium zwischen 10 und $100 \mathrm{mg} \mathrm{RbJ}$ pro Liter.

Bei Anwendung kleiner Mengen Kalium und Uran herrschten die Vasoconstrictoren vor; bei hohen Dosen Kalium (300 mg KCl pro Liter und mehr) traten die Vasodilatatoren nach Nervenreizung in den Vordergrund.

Fügt man dem Nullgemische abwechselnd bestimmte Mengen eines $\alpha$ - und $\beta$-Strahlers zu, so ist man imstande, ein Gemisch zusammen zu stellen, bei dessen Anwendung die Reizbarkeit verschwindet. Zufügung des $\alpha$ - oder $\beta$-Strahlers allein läßt diese sofort wiederkehren, wie es beim Nullgemische der Fall war. So kann man eine ganze Reihe „Gleich- 
616 K. T. A. Halbertsma: Über den Einfluß einzelner radioaktiver Elemente

gewichtsgemische" auffinden zwischen einerseits $K$ und $\mathrm{Rb}$, andererseits $U$ and Th. Die untenstehende Abb. 1 zeigt den Verlauf der „Gleichgewichtslinien", die man durch die Gleichgewichtspunkte legen kann.

Stree $\left.f^{1}\right)$ hat für das Froschherz mittels Fluorescein (100 mg pro Liter) eine Gleichgewichtsverschiebung für das K/U-Gleichgewicht zeigen können. Extra Kalium mußte zugefügt werden, damit das Gleichgewicht sich wieder herstellte. In unserem Fall ließ sich diese Tatsache für die Gleichgewichtsgemische $60 \mathrm{~K} / 15 \mathrm{U}, 100 \mathrm{~K} / 25 \mathrm{U}, 200 \mathrm{~K} / 50 \mathrm{U}$ feststellen; die Mengen Kalium, die in diesen Fällen bzw. hinzugefügt werden mußten, sind auf der nebenstehenden Tabelle mittels Zahlen neben den betreffenden Gleichgewichten angegeben worden.

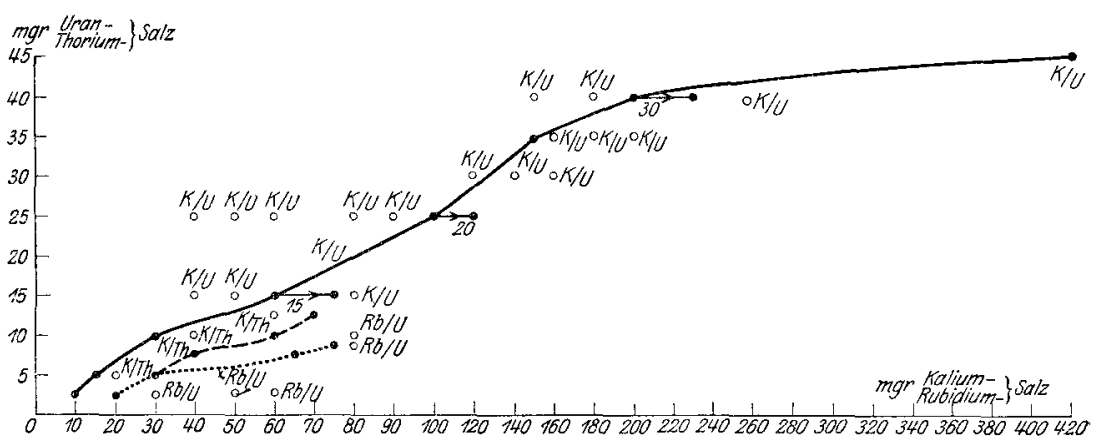

Abb. 1. Übersicht der angewandten Mengen der radioaktiven Salze. $-K / U$ Gleichgewichtslinie, $-\cdots=K / T H, \cdots \cdots=R b / U, 00000=$ Reizbarkeit.

\section{Die Anwendung von Hormonen.}

Es erschien mir interessant, in Zusammenhang mit den bisherigen Ergebnissen meiner Untersuchung den Einfluß einzelner Stoffe kennenzulernen, die in vivo die Gefäßweite überwiegend beherrschen, nämlich des Adrenalins, des Histamins und des Cholins. Weil ich die makroskopische (Tropfenzähl-)Methode verwendete, sollte des bekannten Einflusses dieser Stoffe wegen an erster Stelle dafür gesorgt werden, daß die anzuwendenden Konzentrationen an sich die Gefäßweite nicht änderten. Deswegen wurden zunächst Durchströmungen lediglich mit Hormonenlösungen ausgeführt und die brauchbaren Konzentrationen nachgespürt.

Selbstverständlich hatte ich hierbei den Ergebnissen der Literatur Rechnung zu tragen. Der Einfluß der chemischen Reaktion auf die Wirkung des Adrenalins z. B. ist gezeigt worden von Alday-Redonnet ${ }^{2}$ ),

1) Onderz. Physiol. Laborat. Utrecht. 5 de Reeks 19, 1.

$\left.{ }^{2}\right)$ Biochem. Zeitschr. 120, 306. 1920. 
Synder und Campell ${ }^{1}$ ), Schmidt ${ }^{2}$ ) und P.Heymann ${ }^{3}$ ), auf die Wirkung des Cholins von $W$. Teschendorff ${ }^{4}$ ).

Die Bedeutung der Metallionen (K, Ca, Na) für die Adrenalinwirkung ist von Schmidt (l. c.) und Alday-Redonnet (l. c.) dargestellt worden und für die Acetylcholinwirkung von W. Teschendorff (1. c.).

Unsere Versuche ergaben folgendes:

Das Adrenalin ließ in der Konzentration 1:20 Millionen die Gefäßweite während einiger Stunden unbeeinflußt, Durchströmung mit 1:10 Millionen Adrenalin ließ bald die Gefäße sich verengern, bald die Gefäße unverändert. Anwendung von stärkeren Lösungen rief immer Gefäßverengerung zum Vorschein. Die Lösungen waren mittels Verdünnung des Parke-Davis-Präparates 1: 1000 hergestellt worden.

Histaminlösungen von der Konzentration 1:1000 000, 1:500 000, 1: 330000 ließen die Gefäßweite im allgemeinen unverändert; sie waren mittels Lösung von Ergamintabletten-Wellcome $(0,03 \mathrm{~g}=\mathbf{0 , 0 1} \mathrm{g}$ Histamin) angefertigt worden. Das Acetylcholin ändert in der Konzentration 1: 2,5 Million die Gefäßweite nicht, bei Anwendung von stärkeren Lösungen zogen die Gefäße sich zusammen, eine Gefäßerweiterung wurde nicht beobachtet. Der Stoff wurde hergestellt aus Cholin und EssigsäureAnhydrid nach einer Methode, die mir von le Hueux (Pharmakologisches Institut der R.-U. Utrecht) mitgeteilt wurde, für dessen freundliche Hilfe meinen besten Dank!

Vor dem Anfang jeder Versuchsreihe wurden die Hormonenlösungen immer frisch bereitet.

Beim Nachspüren des Einflusses der Hormonen auf die vasomotorische Erregbarkeit wurden anfänglich die oben angedeuteten Konzentrationen dem Nullgemisch zugefügt.

Das Adrenalin erwies sich imstande, die verschwundene Erregbarkeit, sowohl beim Sommer- wie beim Winternullgemisch wieder auszulösen. Experiment Nr. 4. $11^{\mathrm{h}} 10^{\prime}$. Anfang der Durchströmung mit Sommernullgemisch (3 mg Uran pro Liter). Tropfenzahl pro Minute $=10$.

$11^{\mathrm{h}} 25^{\prime}$. Nervenreizung (Spulendistanz $=26 \mathrm{~cm}$ ). Tropfenzahl in der Minute unmittelbar vor der Reizung $=9$. Tropfenzahl in der Minute unmittelbar nach der Reizung $=9$.

$11^{\mathrm{h}} 25^{\prime}$ bis $11^{\mathrm{h}} 30^{\prime}$. Durchströmung mit kaliumloser Ringerlösung.

$11^{\mathrm{h}} 30^{\prime}$. Anfang der Durchströmung mit dem Nullgemisch + $0,05 \mathrm{mg}$ Adrenalin pro Liter. Tropfenzahl pro Min. $=14$.

$11^{\mathrm{h}} 40^{\prime}$. Nervenreizung (Spulendistanz $=26 \mathrm{~cm}$ ). Tropfenzahl in der Minute unmittelbar vor der Reizung $=14$. Tropfenzahl in der Minute unmittelbar nach der Reizung $=13$.

1) Americ. journ. of physiol. 54, 149. 1920.

$\left.{ }^{2}\right)$ Arch. f. exp. Pathol. u. Pharmakol. 89, 44. 1921.

3) Ibid. 90, 118. 1921.

4) Biochem. Zeitschr. 118, 267. 1921. 
Wurde nun diesem Gemische Kalium in geeigneter Menge (10 mg $\mathrm{KCl}$ pro Liter) zugefügt, so verschwand die zum Vorschein gerufene Reizbarkeit wieder sofort. Diese Tatsache scheint mir, in Zusammenhang mit einem anderen Ergebnisse, das später weiter erörtert werden soll, einen Fingerzeig zu geben, welchem Umstande die Rückkehr der Reizbarkeit bei Durchströmung von (Nullgemisch + Adrenalin) zu verdanken sei.

Drei Möglichkeiten liegen hier meines Erachtens vor: 1. das Uran oder Kalium löst jetzt dank der Anwesenheit des Adrenalins einen Erregbarkeitszustand aus (Sensibilisation); 2. das Adrenalin löst selbst die Erregbarkeit wieder aus; 3 . die sub 1 und 2 genannten Stoffe lösen zusammen die Erregbarkeit wieder aus.

Folgendes Experiment scheint für die sub 1 genannte Möglichkeit zu sprechen: Bei Durchströmung mit 1,5 mg Uransalz pro Liter wurde vasomotorische Erregbarkeit festgestellt und nachdem Adrenalin (1: 20 Millionen) zugefügt worden war, war dieser Zustand erhalten geblieben, wie aus der Gefäßreaktion hervorging. Hinzufügung von $\mathbf{5} \mathbf{~ m g}$ $\mathrm{KCl}$ pro Liter brachte diese zum Verschwinden.

Es war also ein Gemisch zusammengestellt worden aus zwei radioaktiven Elementen und Adrenalin, das dieselbe Eigenschaft hinsichtlich der vasomotorischen Erregbarkeit hatte wie das Nullgemisch. Ausschaltung des Adrenalins ließ sofort die Erregbarkeit wieder zurückkehren, welche durch Zufügung von $1 \mathrm{mg}$ Uransalz pro Liter sofort wieder aufgehoben wurde. Das Adrenalin macht also eine kleinere Menge Uransalz bei Anwesenheit von Kalium ebenso stark wirkend wie eine größere, i. c. das Nullgemisch. Dieses Ergebnis scheint mir also wohl ein Beweis zugunsten der erstgenannten Möglichkeit zu sein.

Auch beim Acetylcholin verschwand nach Zufügung der geeigneten Konzentration dieses Stoffes am Nullgemische der Unreizbarkeitszustand der Vasomotoren. Wurde dem Gemische Kalium zugefügt, so blieb die Erregbarkeit stets erhalten; nur Uran war imstande, in geeigneter Menge diese wieder zum Schwinden zu bringen. Im letzten Falle kehrte nach Zufügung einer kleinen Dosis des radioaktiven Salzes ( $\mathrm{K}$ oder $\mathrm{U}$ ) die verschwundene Reizbarkeit wieder zurück.

Experiment Nr. 5. $10^{\mathrm{h}} 45^{\prime}$. Anfang der Durchströmung mit dem Nullgemisch (2 mg Uransalz pro Liter). Tropfenzahl pro Minute $=12$,

$11^{\mathrm{h}} 00^{\prime}$. Nervenreizung (Spulendistanz $=26 \mathrm{~cm}$ ) Tropfenzahl unmittelbar vor und nach der Reizung $=12$ pro Minute.

$11^{\mathrm{h}} 00^{\prime}$ bis $11^{\mathrm{h}} 15^{\prime}$. Durchströmung mit kaliumloser Ringerlösung. 


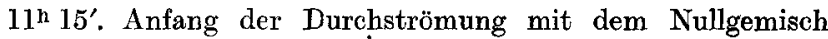
$+0,5 \mathrm{mg}$ Acetylcholin pro Liter. Tropfenzahl in der Minute $=9$.

$11^{\mathrm{h}} 25^{\prime}$. Nervenreizung (Spulendistanz $=26 \mathrm{~cm}$ ). Tropfenzahl in der Minute vor der Reizung $=9$. Tropfenzahl in der Minute nach der Reizung $=6$.

$11^{\mathrm{h}} 25^{\prime}$ bis $11^{\mathrm{h}} 35^{\prime}$. Durchströmung mit kaliumloser Ringerlösung.

$11^{\mathrm{h}} 35^{\prime}$. Anfang der Durchströmung mit (3 mg Uransalz $+0,5 \mathrm{mg}$ Acetylcholin pro Liter).

$11^{\mathrm{h}} 50^{\prime}$. Nervenreizung (Spulendistanz $=26 \mathrm{~cm}$ ). Tropfenzahl unmittelbar vor der Reizung $=6$ in der Minute. Tropfenzahl unmittelbar nach der Reizung $=7$ in der Minute.

$11^{\text {h }} 50^{\prime}$ bis $11^{\text {h }} 55^{\prime}$. Kaliumlose Ringerlösung.

$11^{\text {h }} 55^{\prime}$. Anfang der Durchströmung mit $(3,5 \mathrm{mg}$ Uransalz $+0,5 \mathrm{mg}$ Acetylcholin pro Liter).

$12^{\mathrm{h}} 05^{\prime}$. Nervenreizung (Spulendistanz $=26 \mathrm{~cm}$ ). Tropfenzahl unmittelbar vor und nach der Reizung $=7$ in der Min.

Das Histamin verhält sich hinsichtlich der durch Radioaktivität beeinflußten vasomotorischen Reizbarkeit wie das Adrenalin. Genau wie dort war das Kalium hier (gleichwohl in größerer Menge $=20 \mathrm{mg}$ $\mathrm{KCl}$ pro Liter) imstande, die durch Zufügung des Hormones ausgelöste Reizbarkeit wieder zum Schwinden zu bringen. Uran erwies sich auch hier ohne Wirkung. Erneute Zufügung von geringen Dosen Kalium oder Uran brachte die Reizbarkeit wieder zum Vorschein.

Experiment Nr. 6. $3^{\text {h }} 40^{\prime}$. Anfang der Durchströmung mit $(20 \mathrm{mg} \mathrm{KCl}+3 \mathrm{mg}$ $\mathrm{UO}_{2}\left(\mathrm{NO}_{3}\right)_{2}+3 \mathrm{mg}$ Histamin) pro Liter.

$3^{\mathrm{h}} 50^{\prime}$. Nervenreizung (Spulendistanz $=30 \mathrm{~cm}$ ). Tropfenzahl in der Minute unmittelbar vor der Reizung $=\mathbf{9}$. Tropfenzahl in der Minute unmittelbar nach der Reizung $=9$.

$3^{\text {h }} 55^{\prime}$. Dem Gemisch von $3^{\text {h }} 40^{\prime}$ werden $2 \mathrm{mg}$ Uransalz pro Liter zugefügt. Anfang der Durchströmung.

$4^{\mathrm{h}} 05^{\prime}$. Nervenreizung (Spulendistanz $=30 \mathrm{~cm}$ ). Tropfenzahl in der Minute unmittelbar vor der Reizung $=8$. Tropfenzahl in der Minute unmittelbar nach der Reizung $=7$.

$4^{\text {h }} 10^{\prime}$. Dem Gemisch von $3^{\text {h }} 55^{\prime}$ werden $20 \mathrm{mg} \mathrm{KCl}$ zugefügt. Tropfenzahl zu Anfang der Durchströmung in der Minute $=7$.

$4^{\text {h }} 20^{\prime}$. Nervenreizung (Spulendistanz $=30 \mathrm{~cm}$ ). Tropfenzahl unmittelbar vor und nach der Reizung $=7$.

Es hat sich weiter ergeben, daß Zufügung von geeigneten Hormonenmengen zu Gemischen von Kalium und Uran, bei deren Anwendung die vasomotorische Erregbarkeit erhalten bleibt, imstande ist diese aufzuheben. Für das Adrenalin und das Histamin konnte dies für ungefähr dieselben Mengen gezeigt werden. Das Acetylcholin zeigte sich in dieser Hinsicht refraktär. 
620 K. T. A. Halbertsma: Über den Einfluß einzelner radioaktiver Elemente

Wurde nachher einer der im Gemisch vorhandenen wirksamen Stoffe aufs neue zugefügt, so trat die Reizbarkeit wieder zum Vorschein. Auf Grund dieses Verhaltens nannte ich derartige Gemische ,Adrenalin“. bzw. „Histamin-Gleichgewichtsgemische“.

Schließlich untersuchte ich den Einfluß der Hormone auf einzelne Gleichgewichtsgemische:

$$
\frac{4 \mathrm{mg} \text { Uransalz }}{15 \mathrm{mg} \mathrm{KCl}} \text { pro Liter und } \frac{7,5 \mathrm{mg} \text { Uransalz }}{30 \mathrm{mg} \mathrm{KCl}} \text { pro Liter. }
$$

Auch hier trat die Reizbarkeit nach Zufügung des Hormons sofort wieder zum Vorschein, aber offenbar in den verschiedenen Fällen nicht in derselben Weise. Wurde im Fall von Adrenalin und Histamin eine geeignete Menge Kalium hinzugefügt ( $5 \mathrm{mg} \mathrm{KCl}$ pro Liter), so verschwand die zum Vorschein getretene vasomotorische Erregbarkeit wieder sofort. Dagegen war es im Falle von Acetylcholin nur das Uran, das diese Fähigkeit in geeigneter Menge (1,5 mg Uransalz pro Liter) zeigte.

Das radioaktive Gleichgewicht wurde also bei Anwendung von Adrenalin und Histamin nach der Seite des Urans, bei Anwendung von Acetylcholin nach der des Kaliums verschoben.

Wie aus der folgenden Abbildung hervorgeht, waren die Mengen Kalium bzw. Uran, die zur Wiederherstellung des GJeichgewichtes in

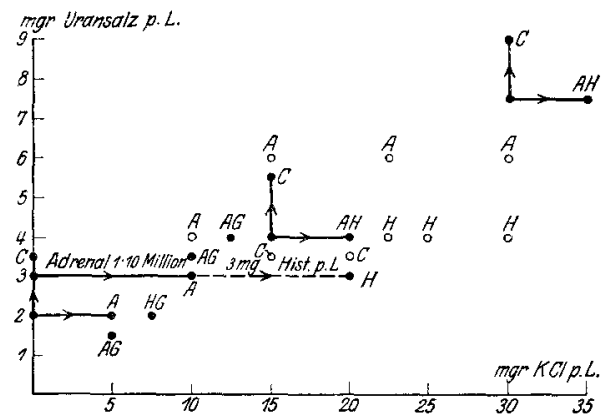

Abb. 2. Übersicht der Hormonenwirkung auf einzelne radio-aktive Gleichgewichtsgemische. $\ldots \ldots \ldots A=$ Gleichgewichts-(Nullgemisch-)verschiebung nach Adrenalinzufügung.

$\ldots \ldots+C=" \quad " \quad " \quad "$ " Acetylcholinzufügung.

$\ldots \ldots \cdots A G=$ Adrenalin-Gleichgewichtsgemisch.

$\ldots \ldots H G=$ Histamin-

o00000 A $=$ Reizbarkeit bei Anwendung der angegebenen Mengen K u. U + Adrenalin. $000000 \mathrm{H}=$ " " " " ", K u. U + Histamin. $000000 \mathrm{C}=\quad, \quad, \quad, \quad, \quad, \quad, \quad$, $\quad, \quad$ K u. U + Acetylchol.

diesen Fällen benötigt sind, unabhängig von der Höhe des untersuchten Gleichgewichtes.

Die Verschiebung des Gleichgewichtes geschah in demselben Sinne, als früher für das Nullgemisch festgestellt worden war. Dieser Befund 
scheint darauf hinzudeuten, daß das Nullgemisch von derselben Art ist wie die untersuchten radioaktiven Gleichgewichtsgemische. Diese Angelegenheit soll im folgenden Abschnitt eingehender besprochen werden.

\section{Der Angriffspunkt der besprochenen Wirkungen.}

Krogh ${ }^{1}$ ) hat neulich eine Methode beschrieben zur Untersuchung der Wirkung lokaler mechanischer Reize auf die Gefäße der Froschschwimmhaut. Wir haben diese Methode mit Vorteil in unserem Falle angewandt.

Während Durchströmung mit dem Nullgemisch wurde die Schwimmhaut des Frosches unter dem Mikroskop (Leitz Oc. 3 Obj. 2) bei schwacher Vergrößerung (45 mal) beobachtet. Einige Minuten nach der Aufstellung wurde einige Male mit einem Druckhaar (20 mg) entlang einem größeren Gefäße gestrichen. Nach ungefähr einer Minute zog sich das Gefäß deutlich zusammen. Dieser Zustand blieb 1-2 Minuten bestehen, dann wurde die Verengerung aufgehoben und die normale Gefäßweite wieder erreicht. Die angrenzenden Capillaren zeigten in diesem Versuche keine Lumenveränderung.

Bei Wiederholung des Versuches während faradischer Reizung des Nerven zog sich das Gefäß nur nach Berührung mit dem Druckhaar ein wenig zusammen; während es sonst bei mikroskopischer Beobachtung während der elektrischen Reizung keine Lumenveränderung zeigte. Auch jetzt wurden an den angrenzenden Capillarendurchschnitten gar keine Veränderungen beobachtet.

Die Gefäßwand war also imstande, während faradischer Reizung bei Durchströmung mit dem Nullgemisch nur auf mechanische Reizung zu reagieren; die contractilen Elemente waren offenbar funktionsfähig geblieben und die Nervenfasern leiteten anscheinend die zugeführten Reize nicht in derselben Weise über als es bei Anwendung von ,aktiven“" Gemischen der Fall war.

Daß dies wirklich der Fall war, zeigte folgender Versuch:

Bei Durchströmung mit (4 mg Uransalz $+15 \mathrm{mg} \mathrm{KCl}$ ) pro Liter folgte auf der faradischen Reizung eine leichte Gefäßkontraktion; die Gefäßverengerung nach mechanischer Reizung war stärker als in den früher beschriebenen Versuchen.

Hier übten die nervösen Elemente einen bedeutenden Einfluß auf die Gefäßwand aus, die contractilen Elemente hatten jedoch ihre Funktion behalten.

Bei Anwendung von Adrenalin und Histamin zeigte sich das Verhalten der vasomotorischen Reizbarkeit wie bei Durchströmung mit

1) Journ. of physiol. 55.1921. 
dem letztgenannten aktiven Gemisch; die faradische und mechanische Reizbarkeit waren also gleichzeitig hier vorhanden.

Es liegt also auf der Hand anzunehmen, daß in denjenigen Fällen, wo faradische Reizbarkeit der Vasomotoren besteht, die Reizübertragung an der Übergangsstelle zwischen contractilen und nervösen Elementen, d. h. die Synapse, stattfindet.

Beim Nullgemisch und bei den Gleichgewichtsgemischen, wo die faradische Reizbarkeit aufgehoben ist, ist meiner Meinung nach die Aufhebung der Reizleitung an dieser Stelle zu suchen. Noch einen anderen Schluß darf man meines Erachtens aus den hier im vorliegenden beschriebenen Ergebnissen ziehen, nämlich, daß das Nullgemisch den Gleichgewichtsgemischen zuzurechnen ist. Nicht nur die Ähnlichkeit zwischen beiden Gruppen von Gemischen hinsichtlich der Wiederherstellung der Reizbarkeit durch radioaktive Salze und Hormone, aber auch der mikroskopische Befund, deutet auf eine Identität beider Gruppen hin. Das Nullgemisch ist infolge dieser Auffassung also als ,Minimum-Gleichgewichtsgemisch" zu betrachten und es liegt auf der Hand, die Menge Uransalz (2-4 $\mathrm{mg}$ pro Liter) einerseits und die in der Ausströmungsflüssigkeit anwesenden Mengen Kalium andererseits als die beiden Antagonisten, in diesem Falle zu betrachten. Der Parallelismus zwischen der gefundenen wechselnden Mengen Kalium und Uran in den verschiedenen Jahreszeiten scheinen mir eine bedeutende Stütze für diese Auffassung zu sein.

\section{Zusammenfassung.}

1. Vermittelst eines Kunstgriffes - Zufügung von 2-3 mg Uransalz im Sommer, 3-4 mg Uransalz im Winter - ist es möglich, die faradische Reizbarkeit der Vasomotoren beim Frosch, die sonst bei Durchströmung mit kaliumloser Ringer schen Flüssigkeit bestehen bleibt, zum Schwinden zu bringen.

2. Es ist möglich, durch Zufügung geeigneter Mengen radioaktiven Salzes an diesem Gemische die Reizbarkeit wieder zum Vorschein treten zu lassen.

3. Durch Zufügung geeigneter $\alpha$ - und $\beta$-Strahler ist es möglich, eine ganze Reihe Gleichgewichtsgemische zusammen zu stellen: die Reizbarkeit ist in diesen Fällen verschwunden. Das sub 1 genannte Gemisch - Nullgemisch - stellt das Minimumgleichgewichtsgemisch dar. Zerstörung des Gleichgewichtes hat sofortige Rückkehr der Reizbarkeit zur Folge.

4. Die Gleichgewichtslinien stellen anfangs ziemlich steil aufsteigende, allmählich mehr horizontal verlaufende Linien dar. Die Abszisse wird von den leichten Metallionen, die Ordinate von den schweren 
Metallionen gebildet. Diese Linien wurden bei gleichzeitiger Durchströmung mit Kalium/Uran, Kalium/Thorium und Rubidium/Uran festgestellt.

5. Die Hormone Adrenalin, Histamin und Cholin lassen nach Zufügung am Nullgemische und die höheren Gleichgewichtsgemische sofort die Reizbarkeit wieder zurückkehren.

6. Das Cholin verstärkt den Einfluß der leichten radioaktiven Ionen $(\mathrm{K}, \mathrm{Rb})$; das Adrenalin und Histamin den der schweren Ionen $\left(\mathrm{UO}_{2}\right)$. Zufügung einer geeigneten Menge der antagonistischen Ionen läßt die Reizbarkeit wieder verschwinden; werden aufs neue die ursprünglichen Stoffe zugefügt, so kehrt die Reizbarkeit sofort wieder zurück.

7. Die Wirkung der radioaktiven Salze auf die vasomotorischen Reizbarkeit hat ihren Angriffspunkt in der Synapse. 